\title{
Astrometric accuracy of phase-referenced observations with the VLBA and EVN
}

\author{
N. Pradel ${ }^{1}$, P. Charlot ${ }^{1}$, and J.-F. Lestrade ${ }^{2}$ \\ 1 Observatoire de Bordeaux (OASU) - CNRS/UMR 5804, BP 89, 33270 Floirac, France \\ e-mail: pradel@obs.u-bordeaux1.fr \\ 2 Observatoire de Paris - CNRS/UMR 8112, 61 avenue de l'Observatoire, 75014 Paris, France
}

Received 8 March 2005 / Accepted 4 January 2006

\section{ABSTRACT}

\begin{abstract}
Astrometric accuracy of complex modern VLBI arrays cannot be calculated analytically. We study the astrometric accuracy of phasereferenced VLBI observations for the VLBA, EVN and global VLBI array by simulating VLBI data for targets at declinations $-25^{\circ}$, $0^{\circ}, 25^{\circ}, 50^{\circ}, 75^{\circ}$ and $85^{\circ}$. The systematic error components considered in this study are calibrator position, station coordinate, Earth orientation and troposphere parameter uncertainties. We provide complete tables of the astrometric accuracies of these arrays for a source separation of $1^{\circ}$ either along the right ascension axis or along the declination axis. Astrometric accuracy is $50 \mu$ as at mid declination and is $300 \mu$ as at low $\left(-25^{\circ}\right)$ and high $\left(85^{\circ}\right)$ declinations for the VLBA and EVN. In extending our simulations to source separations of $0.5^{\circ}$ and $2^{\circ}$, we establish the formula for the astrometric accuracy of the VLBA: $\Delta_{\alpha \cos \delta, \delta}=\left(\Delta_{\alpha \cos \delta, \delta}^{1^{\circ}}-14\right) \times d+14(\mu$ as $)$ where $\Delta_{\alpha \cos \delta, \delta}^{1^{\circ}}$ is the astrometric accuracy for a separation $d=1^{\circ}$ provided in our tables for various declinations and conditions of the wet troposphere. We argue that this formula is also valid for the astrometric accuracy of the EVN and global VLBI array.
\end{abstract}

Key words. astrometry - techniques: high angular resolution - techniques: interferometric

\section{Introduction}

Very Long Baseline Interferometry (VLBI) narrow-angle astrometry pioneered by Shapiro et al. (1979) makes use of observations of pairs of angularly close sources to cancel atmospheric phase fluctuations between the two close lines of sight. In this initial approach, the relative coordinates between the two strong quasars 3C 345 and NRAO512 and other ancilliary parameters were adjusted by a least-squares fit of the differenced phases after connecting the VLBI phases for both sources over a multi-hour experiment. Then, Marcaide \& Shapiro $(1983,1984)$ made the first phase-referenced map where structure and astrometry were disentangled for the double quasar $1038+528 \mathrm{~A}$ and $\mathrm{B}$. Both of these experiments demonstrated formal errors at the level of a few tens of microarcseconds or less in the relative angular separation between the two sources.

Another approach was designed to tackle faint target sources by observing a strong reference source (quasar) to increase the integration time of VLBI from a few minutes to a few hours (Lestrade et al. 1990). This approach improves the sensitivity by the factor

$\sqrt{N_{\mathrm{b}} \times \frac{T_{\mathrm{int}}}{T_{\text {scan }}}}$

where $N_{\mathrm{b}}$ is the number of VLBI baselines, $T_{\text {int }}$ is the extended integration time permitted by phase-referencing (several hours) and $T_{\text {scan }}$ is the individual scan length (a few minutes). As this factor is very large (e.g. $>50$ for the 45 baselines of the Very Long Baseline Array), faint target sources can be detected and their positions can be concomitantly measured with high precision.

In the approach above, the VLBI phases of the strong reference source are connected, interpolated in time and differenced with the VLBI phases of the faint source that do not need to be connected. The differenced visibilities are then inverted to produce the map of the brightness distribution of the faint target source and its position is determined by reading directly the coordinates of the map peak which are relative to the a priori reference source coordinates. The map is usually highly undersampled but suffices for astrometry. This mapping astrometry technique is implemented in the SPRINT software (Lestrade et al. 1990) and a similar procedure is also used within the NRAO AIPS package to produce phase-referenced VLBI maps with absolute source coordinates on the sky.

While phase-referencing in this way is efficient, it still provides no direct positional uncertainty as does least-squares fitting of differenced phases (Shapiro et al. 1979). In order to circumvent this problem, we have developed simulations to evaluate the impact of systematic errors in the derived astrometric results. Such simulations have been carried out for of a pair of sources observed with the Very Long Baseline Array (VLBA) and the European VLBI Network (EVN) at various declinations and angular separations. Systematic errors in station coordinates, Earth rotation parameters, reference source coordinates and tropospheric zenith delays were studied in turn. The results of the simulations are summarized below in tables that indicate positional uncertainties when considering these systematic errors either separately or altogether. Such tables can be further interpolated to determine the accuracy of any full-track experiment with the VLBA and EVN.

Our study includes atmospheric fluctuations caused by the turbulent atmosphere above all stations. These fluctuations have been considered uniform and equivalent to a delay rate noise of $0.1 \mathrm{ps} / \mathrm{s}$ for all stations. The impact of these fluctuations is limited if the antenna switching cycle between the two sources is fast enough. The phase structure function measured at $22 \mathrm{GHz}$ 
above the VLA by Carilli \& Holdaway (1999) provides prescriptions on this switching time. At high frequency, it can be as short as $10 \mathrm{~s}$, as e.g. in Reid et al. (2003) who carried out precise $43 \mathrm{GHz}$ VLBA astrometric observations of Sgr A* at a declination of $-28^{\circ}$. Switching time in more clement conditions is typically a few minutes at $8.4 \mathrm{GHz}$ for northern sources.

A few applications of mapping astrometry are the search for extra-solar planets around radio-emitting stars (Lestrade et al. 1994), the determination of the Gravity Probe B guide star proper motion (Lebach et al. 1999), the determination of absolute motions of VLBI components in extragalactic sources, e.g. in compact symetric objects (Charlot et al. 2005) or core-jet sources (Ros et al. 1999), probing the jet collimation region in extragalactic nuclei (Ly et al. 2004), pulsar parallax and proper motion measurements (Brisken et al. 2002) and the determination of parallaxes and proper motions of maser sources in the whole Galaxy as planned with the VERA project (Kawaguchi et al. 2000; Honma et al. 2000).

\section{Method}

As indicated in e.g. Thompson et al. (1986), the theoretical precision of astrometry with the interferometer phase is

$\sigma_{\alpha, \delta}=\frac{1}{2 \pi} \frac{1}{S N R} \frac{\lambda}{B}$

where $S N R$ is the signal-to-noise ratio of the observation, $\lambda$ is the wavelength and $B$ is the baseline length projected on the sky. For observations with the VLBA $(B \sim 8000 \mathrm{~km}), \lambda=3.6 \mathrm{~cm}$, and a modest $S N R$ of 10, this theoretical precision is breathtakingly $\sim 15 \mu$ as. Although a single observation of the target yields an ambiguous position, multiple observations over several hours easily remove ambiguities even with a sparse $u-v$ plane coverage (Lestrade et al. 1990).

While the theoretical precision above might be regarded as the potential accuracy attainable for the VLBI, systematic errors in the model of the phase limit narrow-angle astrometry precision to roughly ten times this level in practice (Fomalont et al. 1999). An analytical study of systematic errors in phasereferenced VLBI astrometry over a single baseline is given in Shapiro et al. (1979) and it shows that all systematic errors are scaled by the source separation. Another error analysis in such differential VLBI measurements can be found in Morabito (1984). However, for modern VLBI arrays with 10 or more antennae, the complex geometry makes the analytical approach intractable. For this reason, we have estimated such systematic errors by simulating VLBI visibilities and inverting them for a range of model parameters (station coordinates, reference source coordinates, Earth Orientation parameters, and tropospheric dry and wet zenith delays) corresponding to the expected errors in these parameters.

The visibilities were simulated for a pair of sources at declinations $-25^{\circ}, 0^{\circ}, 25^{\circ}, 50^{\circ}, 75^{\circ}, 85^{\circ}$ and with angular separations $0.5^{\circ}, 1^{\circ}$ and $2^{\circ}$ for the VLBA, EVN and global VLBI array (VLBA+EVN). For each of these cases, we simulated visibilities every $2.5 \mathrm{~min}$ from source rise to set (full track) with a lower limit on elevation of $7^{\circ}$. The adopted flux for each source (calibrator and target) was $1 \mathrm{Jy}$ to make the phase thermal noise negligeable in our simulations. For applications to faint target sources, one should combine the corresponding thermal astrometric uncertainty (Eq. (1)) with the systematic errors derived below. The simulated visibilities were then inverted using uniform weighting to produce a phase-referenced map of the target source and estimate its position. This operation was repeated
100 times in a Monte Carlo analysis after varying slightly the parameters of the model based on errors drawn from a Gaussian distribution with zero-mean and plausible standard deviation. We report the rms of the differences found between the known a priori position of the target source and the resulting estimated positions as a measure of the corresponding systematic errors for each of the above cases. We have adopted the usual astrometric frequency of $8.4 \mathrm{GHz}$ for this analysis.

\section{Phase model used in simulation}

The phase delay and group delay in VLBI are described in Sovers et al. (1998). The phase $\phi=v \tau$ at frequency $v$ is related to the interferometer delay

$\tau=\tau_{\mathrm{g}}+\tau_{\text {trop }}+\tau_{\text {iono }}+\tau_{\mathrm{R}}+\tau_{\text {struc }}+\tau_{\text {clk }}$

Specifically, the geometric delay is:

$\tau_{\mathrm{g}}=[P][N][E O P] \frac{\boldsymbol{b} \cdot \boldsymbol{k}}{c}$

with the precession matrix $[P]$, the nutation matrix $[N]$, the Earth Orientation Parameters matrix [EOP], the baseline coordinates $\boldsymbol{b}$ in the terrestrial frame, the source direction coordinates $\boldsymbol{k}$ computed with source right ascension and declination in the celestial frame. The "retarded baseline correction" to account for Earth rotation during elapsed time $\tau_{\mathrm{g}}$ must also be modelled (Sovers et al. 1998). The differential tropospheric delay $\tau_{\text {trop }}$ between the two stations is computed with a static tropospheric model and the simple mapping function $1 / \sin E$ (where $E$ is the source elevation at station) to transform the zenith delay into the lineof-sight delay at each station. The differential ionospheric phase delay $\tau_{\text {iono }}=-k \mathrm{TEC} / v^{2}$ is related to the total electronic content TEC in the direction of the source at each station. The General Relativity delay $\tau_{\mathrm{R}}$ takes into account light propagation travel time in the gravitational potential of the Sun. The source structure contribution $\tau_{\text {struc }}$ can be computed according to the model by Charlot (1990) but was not included in our simulations which are for point sources. The clock delay $\tau_{\text {clk }}$ cancels in differenced VLBI phases.

The model above is that implemented in the SPRINT software used for our simulations. It is thought to be complete for narrow-angle astrometry and additional refinements, such as ocean loading, atmospheric loading, etc., would not make difference into our results. We have not studied the ionosphere contribution to systematic errors. The unpredictible nature of the ionosphere makes this task difficult. Calibration of the ionosphere by dual-frequency observations, or over a wide bandwidth at low frequency (Brisken et al. 2002), or simply by observing at high frequency $(>10 \mathrm{GHz})$ where the effect is small, offers solutions to this problem.

\section{Results}

\subsection{VLBA}

The parameter rms errors adopted as plausible for the VLBA phase model are listed in Tables 1 and 2. The reference source coordinate uncertainties $\left(\Delta \alpha_{0} \cos \delta_{0}, \Delta \delta_{0}\right)$ of 1 mas are typical of those in the VLBA Calibrator Survey (Beasley et al. 2002), from which most of the reference sources originate. However, ICRF extragalactic sources have better position accuracies down to 0.25 mas (Ma et al. 1998). We have thus carried out the calculations for both of these cases ( 1 mas and 0.25 mas) and both $\alpha_{0}$ 
Table 1. Adopted rms errors for the source coordinates, VLBA station coordinates and Earth Orientation Parameters in our Monte Carlo simulations.

\begin{tabular}{lc}
\hline \hline Parameters & Errors \\
\hline Source coordinates & $0.25 / 1 \mathrm{mas}$ \\
$\alpha_{0} \cos \delta_{0}$ & $0.25 / 1 \mathrm{mas}$ \\
$\delta_{0}$ & $1-2 \mathrm{~mm}$ \\
\hline Station coordinates & $1-3 \mathrm{~mm}$ \\
$X$ & $1-2 \mathrm{~mm}$ \\
$Y$ & \\
$Z$ & $0.2 \mathrm{mas}$ \\
\hline Earth orientation parameters & $0.2 \mathrm{mas}$ \\
$X_{\mathrm{p}}$ & $0.02 \mathrm{~ms}$ \\
$Y_{\mathrm{p}}$ & $0.3 \mathrm{mas}$ \\
$U T 1-U T C$ & $0.3 \mathrm{mas}$ \\
$\psi \sin \epsilon$ & \\
$\epsilon$
\end{tabular}

Table 2. Dry and wet tropospheric zenith path delays ( $\tau_{\mathrm{dtrp}}$ and $\left.\tau_{\mathrm{wtrp}}\right)$ at the VLBA stations along with the adopted rms errors $\Delta \tau_{\mathrm{dtrp}}$ and $\Delta \tau_{\mathrm{wtrp}}$ in our Monte Carlo simulations.

\begin{tabular}{lcccccc}
\hline \hline Stations & \multicolumn{2}{c}{ Dry trop. } & \multicolumn{3}{c}{ Wet trop. } \\
& & \multicolumn{4}{c}{ Mean } & Max \\
& $\tau_{\text {dtrp }}$ & $\Delta \tau_{\text {dtrp }}$ & $\tau_{\text {wtrp }}$ & $\Delta \tau_{\text {wtrp }}$ & $\tau_{\text {wtrp }}$ & $\Delta \tau_{\text {wtrp }}$ \\
& $(\mathrm{cm})$ & $(\mathrm{cm})$ & $(\mathrm{cm})$ & $(\mathrm{cm})$ & $(\mathrm{cm})$ & $(\mathrm{cm})$ \\
\hline Brewster & 225 & 0.5 & 8 & 2.7 & 13 & 4.3 \\
Fort Davis & 192 & 0.5 & 8 & 2.7 & 15 & 5.0 \\
Hancock & 223 & 0.5 & 9 & 3.0 & 19 & 6.3 \\
Kit Peak & 185 & 0.5 & 6 & 2.0 & 15 & 5.0 \\
Los Alamos & 185 & 0.5 & 6 & 2.0 & 13 & 4.3 \\
Mauna Kea & 149 & 0.5 & 1 & 2.0 & 4 & 2.0 \\
North Liberty & 225 & 0.5 & 10 & 3.3 & 19 & 6.3 \\
Owens Valley & 199 & 0.5 & 5 & 2.0 & 20 & 6.7 \\
Pietown & 176 & 0.5 & 4 & 2.0 & 12 & 4.0 \\
Saint Croix & 213 & 0.5 & 22 & 7.3 & 30 & 10.0 \\
\hline
\end{tabular}

and $\delta_{0}$ have been perturbed by these uncertainties in our simulations. The uncertainties for the station coordinates are from the ITRF2000 frame (Boucher et al. 2004) while those for the Earth Orientation Parameters are from the IERS web site ${ }^{1}$. The adopted dry tropospheric rms error $\Delta \tau_{\text {dtrp }}$ of $0.5 \mathrm{~cm}$ corresponds to 2.5 millibars in atmospheric pressure uncertainty at sea level. Although barometer reading is usually better, the absolute calibration of station barometers is at this level. Uncertainties in the wet tropospheric zenith delay $\tau_{\text {wtrp }}$ derived from temperature and humidity are known to be large (Saastamoinen 1973). Experience makes us believe that a $30 \%$ error is likely on $\tau_{\text {wtrp }}$ and thus we took $1 / 3$ of $\tau_{\text {wtrp }}$ as the plausible rms error $\Delta \tau_{\text {wtrp }}$ with a minimum value of $2 \mathrm{~cm}$. We carried out simulations for both mean and maximum values of wet zenith path delays based on estimates of $\tau_{\text {wtrp }}$ recently derived from multiple VLBA geodetic and astrometric sessions (Sovers 2003). The maximum wet zenith delays and corresponding errors were used to investigate the impact of extreme weather conditions on observations. These values are listed for each VLBA station in Table 2.

We simulated the visibilities of a full $u-v$ track experiment with the VLBA for six declinations between $-25^{\circ}$ and $85^{\circ}$ with a $1^{\circ}$ relative source separation (either oriented in right ascension or in declination). Uniform weighting was applied to the visibilities, resulting in a synthesized beam mainly shaped by the longest baselines. As a test, we have also removed the

1 http://hpiers.obspm. fr/iers/eop/eopc04/EOPCO4.GUIDE (Table 2).
9 baselines smaller than $1500 \mathrm{~km}$ in length out of the 45 baseline array and noted a decrease in systematic errors of $\sim 15 \%$ in a few test cases. Conservatively, we have retained these "short" baselines in our final simulations. This is motivated by the fact that all possible baselines must be kept for sensitivity when observing weak sources. The antenna switching cycle between target and reference sources was set to $2.5 \mathrm{~min}$. The results, however, do not depend critically on this value. It was chosen so that the automatic phase connection routine for the reference source does not discard too much data in the presence of a delay rate error of $0.1 \mathrm{ps} / \mathrm{s}$ (adopted uniformly for all the stations in the simulation). As mentioned previously, we analysed these data simulated with SPRINT using the a priori parameter values perturbed by some errors. We carried out this analysis 100 times for each systematic error component with perturbation errors drawn from Gaussian distributions with zero mean and standard deviations according to the rms errors in Tables 1 and 2. The resulting position of the target was estimated by reading the peak position in each of the 100 phase-referenced maps. The pixel size in the maps was 0.05 mas. This size is small compared to the synthesized beam ( $\sim 1$ mas at $8.4 \mathrm{GHz}$ on $8000 \mathrm{~km}$ baseline) and, hence, the uncertainty in the peak position due to the pixel size is negligeable. This position was determined by fitting a parabola over the full half beam width. This procedure was used in the Hipparcos/VLBI work of Lestrade et al. (1999) and was found to be appropriate. As expected, each position was slightly offset from the map phase center, reflecting the corresponding systematic errors. After substracting the initial perturbation in the calibrator position, we calculated the rms of these 100 relative coordinate offsets $\Delta \alpha \cos \delta$ and $\Delta \delta$ for the adopted $1^{\circ}$ source separation in right ascension or declination. Note that the mean of these 100 coordinate offsets was close to zero in all cases. In Tables 3 and 4, we report the rms astrometric errors for each individual error component along with the total astrometric errors when all model parameters are perturbed together in the simulation. The total errors were derived by considering a 1 mas error in the calibration position.

The wet troposphere systematic error clearly dominates over all the other error components for $\delta \leq 50^{\circ}$ but the calibrator error dominates at higher declinations if its position is not known to better than 1 mas. This behavior was first noted by Shapiro et al. (1979) who derived analytical formulae providing the astrometric errors caused by the calibrator coordinate uncertainties in the case of a single VLBI baseline. A detailed analysis comparing our simulated errors with those obtained from these formulae is given in Appendix A. Other systematic errors, in particular the Earth orientation parameter and the station coordinate errors, are small. In Tables 3 and 4, we note that astrometric errors originating from mean and maximum wet troposphere uncertainties are not drastically different (a ratio of 1.5 at most).

Finally, we have plotted in Fig 1 the distribution of all coordinate offsets $\Delta \alpha \cos \delta$ and $\Delta \delta$ for the $50^{\circ}$ declination target when all perturbation errors are present. For this specific case we have carried out 1000 simulations to refine the binning of the distribution. We have also performed the Pearson test on all distributions and provide the reduced chi-square $\chi_{v}^{2}$ and probability $p$ that such distributions are Gaussian in Table 5. The results of this test show that most of the distributions are not Gaussian with $p$ generally smaller than 0.4 .

\section{2. $E V N$}

We have carried out a similar study for the EVN by simulating full track observations for the 10 stations of the array at 
Table 3. VLBA rms astrometric errors (in $\mu$ as) for a relative source separation $\left(\alpha-\alpha_{0}\right) \cos \delta_{0}=1^{\circ}$. Individual astrometric error contributions from calibrator position, Earth orientation parameter, station coordinate, and dry and wet troposphere uncertainties are given separately, while the last two lines indicate the total astrometric errors when all model parameters are perturbed together.

\begin{tabular}{|c|c|c|c|c|c|c|c|c|c|c|c|c|}
\hline \multirow[b]{3}{*}{ Error component } & \multicolumn{12}{|c|}{ Declination of source } \\
\hline & \multicolumn{2}{|l|}{$-25^{\circ}$} & \multicolumn{2}{|l|}{$0^{\circ}$} & \multicolumn{2}{|l|}{$25^{\circ}$} & \multicolumn{2}{|l|}{$50^{\circ}$} & \multicolumn{2}{|l|}{$75^{\circ}$} & \multicolumn{2}{|l|}{$85^{\circ}$} \\
\hline & $\Delta \alpha \cos \delta$ & $\Delta \delta$ & $\Delta \alpha \cos \delta$ & $\Delta \delta$ & $\Delta \alpha \cos \delta$ & $\Delta \delta$ & $\Delta \alpha \cos \delta$ & $\Delta \delta$ & $\Delta \alpha \cos \delta$ & $\Delta \delta$ & $\Delta \alpha \cos \delta$ & $\Delta \delta$ \\
\hline Calibrator position (1 mas error) & 8 & 7 & 1 & 9 & 8 & 16 & 20 & 26 & 59 & 68 & 196 & 193 \\
\hline Calibrator position ( 0.25 mas error) & 2 & 7 & 1 & 3 & 2 & 5 & 3 & 5 & 12 & 11 & 49 & 50 \\
\hline Earth orientation & 1 & 8 & 1 & 5 & 1 & 6 & 1 & 5 & 1 & 4 & 1 & 4 \\
\hline Antenna position & 2 & 8 & 2 & 4 & 2 & 4 & 2 & 3 & 2 & 3 & 2 & 3 \\
\hline Dry troposphere & 15 & 45 & 9 & 16 & 7 & 9 & 10 & 11 & 18 & 23 & 14 & 16 \\
\hline Wet troposphere (mean) & 53 & 182 & 34 & 57 & 33 & 28 & 31 & 45 & 54 & 72 & 79 & 88 \\
\hline Wet troposphere $(\max )$ & 87 & 219 & 46 & 66 & 42 & 38 & 49 & 56 & 65 & 78 & 81 & 91 \\
\hline Total (mean wtrp) & 60 & 175 & 36 & 50 & 33 & 32 & 37 & 53 & 87 & 103 & 227 & 258 \\
\hline Total (max wtrp) & 85 & 217 & 43 & 74 & 42 & 44 & 46 & 66 & 100 & 117 & 226 & 240 \\
\hline
\end{tabular}

Table 4. VLBA rms astrometric errors (in $\mu$ as) for a relative source separation $\delta-\delta_{0}=1^{\circ}$. Individual astrometric error contributions from calibrator position, Earth orientation parameter, station coordinate, and dry and wet troposphere uncertainties are given separately, while the last two lines indicate the total astrometric errors when all model parameters are perturbed together.

\begin{tabular}{|c|c|c|c|c|c|c|c|c|c|c|c|c|}
\hline \multirow[b]{3}{*}{ Error component } & \multicolumn{12}{|c|}{ Declination of source } \\
\hline & \multicolumn{2}{|c|}{$-25^{\circ}$} & \multicolumn{2}{|l|}{$0^{\circ}$} & \multicolumn{2}{|l|}{$25^{\circ}$} & \multicolumn{2}{|l|}{$50^{\circ}$} & \multicolumn{2}{|l|}{$75^{\circ}$} & \multicolumn{2}{|l|}{$85^{\circ}$} \\
\hline & $\Delta \alpha \cos \delta$ & $\Delta \delta$ & $\Delta \alpha \cos \delta$ & $\Delta \delta$ & $\Delta \alpha \cos \delta$ & $\Delta \delta$ & $\Delta \alpha \cos \delta$ & $\Delta \delta$ & $\Delta \alpha \cos \delta$ & $\Delta \delta$ & $\Delta \alpha \cos \delta$ & $\Delta \delta$ \\
\hline Calibrator position (1 mas error) & 7 & 8 & 1 & 7 & 8 & 11 & 21 & 2 & 59 & 2 & 199 & 1 \\
\hline Calibrator position ( 0.25 mas error) & 2 & 7 & 1 & 3 & 2 & 4 & 5 & 2 & 20 & 1 & 43 & 1 \\
\hline Earth orientation & 5 & 7 & 5 & 3 & 5 & 4 & 4 & 3 & 3 & 1 & 3 & 1 \\
\hline Antenna position & 2 & 9 & 2 & 6 & 2 & 5 & 3 & 3 & 2 & 2 & 2 & 2 \\
\hline Dry troposphere & 17 & 54 & 6 & 19 & 2 & 12 & 5 & 9 & 11 & 9 & 12 & 13 \\
\hline Wet troposphere (mean) & 80 & 272 & 32 & 98 & 11 & 41 & 12 & 32 & 43 & 41 & 60 & 62 \\
\hline Wet troposphere (max) & 112 & 358 & 43 & 114 & 19 & 61 & 17 & 46 & 59 & 55 & 74 & 71 \\
\hline Total (mean wtrp) & 84 & 284 & 30 & 99 & 16 & 42 & 25 & 33 & 81 & 36 & 189 & 67 \\
\hline Total (max wtrp) & 121 & 481 & 44 & 134 & 20 & 56 & 26 & 46 & 92 & 65 & 212 & 74 \\
\hline
\end{tabular}
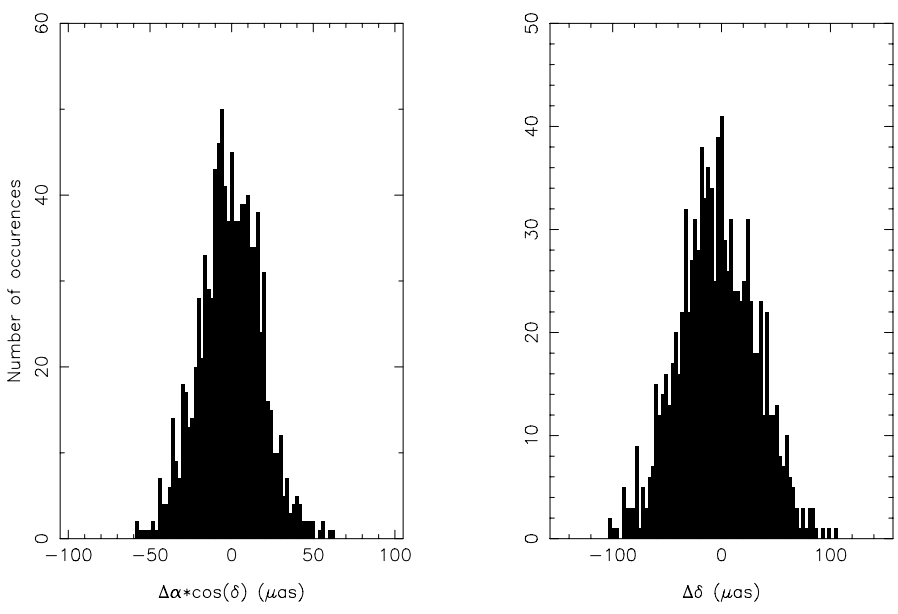

Fig. 1. Distribution of total astrometric errors for a $1^{\circ}$ relative source separation along declination at declination $50^{\circ}$. All perturbating errors (calibrator position, Earth orientation parameters, station coordinates, dry and wet troposphere parameters) are considered together in this simulation.

8.4 GHz. The adopted errors for the reference source coordinates and Earth orientation parameters were identical to those used in the VLBA simulations. Station coordinate errors were similar to the VLBA ones (1-6 mm), with the exception of those for Westerbork which are at the level of $50 \mathrm{~mm}$ (Charlot et al. 2002). The same scheme as that adopted for the VLBA was used
Table 5. Reduced chi-square $\chi_{v}^{2}$ and probability $p$ of Gaussian distribution for the astrometric errors $\Delta \alpha \cos \delta$ and $\Delta \delta$ using the Pearson test.

\begin{tabular}{|c|c|c|c|c|}
\hline \multirow{3}{*}{ Declination } & \multicolumn{4}{|c|}{$\left(\alpha-\alpha_{0}\right) \cos \delta_{0}=1^{\circ}$} \\
\hline & \multicolumn{2}{|c|}{$\Delta \alpha \cos \delta$} & \multicolumn{2}{|c|}{$\Delta \delta$} \\
\hline & $\chi_{v}^{2}$ & $p$ & $\chi_{v}^{2}$ & $p$ \\
\hline$-25^{\circ}$ & 1.51 & $1.43 \times 10^{-2}$ & 2.10 & $9.59 \times 10^{-6}$ \\
\hline $0^{\circ}$ & 1.67 & $1.29 \times 10^{-2}$ & 1.73 & $1.95 \times 10^{-3}$ \\
\hline $25^{\circ}$ & 2.33 & $8.12 \times 10^{-8}$ & 1.97 & $1.12 \times 10^{-3}$ \\
\hline $50^{\circ}$ & 0.97 & 0.511 & 1.18 & 0.309 \\
\hline $75^{\circ}$ & 0.84 & 0.783 & 1.22 & 0.273 \\
\hline \multirow[t]{2}{*}{$85^{\circ}$} & 1.06 & 0.659 & 1.02 & 0.424 \\
\hline & \multicolumn{4}{|c|}{$\left(\delta-\delta_{0}\right)=1^{\circ}$} \\
\hline \multirow[t]{2}{*}{ Declination } & \multicolumn{2}{|c|}{$\Delta \alpha \cos \delta$} & \multicolumn{2}{|r|}{$\Delta \delta$} \\
\hline & $\chi_{v}^{2}$ & $p$ & $\chi_{v}^{2}$ & $p$ \\
\hline$-25^{\circ}$ & 1.19 & 0.286 & 0.76 & 0.885 \\
\hline $0^{\circ}$ & 1.62 & $2.24 \times 10^{-2}$ & 1.35 & $6.01 \times 10^{-2}$ \\
\hline $25^{\circ}$ & 3.22 & $4.62 \times 10^{-4}$ & 1.91 & $5.57 \times 10^{-4}$ \\
\hline $50^{\circ}$ & 2.23 & $5.19 \times 10^{-6}$ & 2.18 & $1.28 \times 10^{-4}$ \\
\hline $75^{\circ}$ & 1.38 & 0.116 & 2.05 & $1.99 \times 10^{-3}$ \\
\hline $85^{\circ}$ & 1.22 & 0.260 & 2.98 & $5.62 \times 10^{-12}$ \\
\hline
\end{tabular}

to define zenith dry and wet tropospheric delay errors at each EVN station and the corresponding values are given in Table 6.

Since the EVN comprises antennas with different sensitivities, each baseline has been weighted by the reciprocal of their noise power equivalent $\sqrt{\mathrm{SEFD}_{1} \times \mathrm{SEFD}_{2}}$ with System 
Table 6. Dry and wet tropospheric zenith path delays $\left(\tau_{\text {dtrp }}\right.$ and $\left.\tau_{\text {wtrp }}\right)$ at the EVN stations along with the adopted rms errors $\Delta \tau_{\text {dtrp }}$ and $\Delta \tau_{\text {wtrp }}$ in our Monte Carlo simulations.

\begin{tabular}{lcccccc}
\hline \hline Stations & \multicolumn{2}{c}{ Dry trop. } & \multicolumn{4}{c}{ Wet trop. } \\
& & \multicolumn{4}{c}{ Mean } & \multicolumn{2}{c}{ Max } \\
& $\tau_{\text {drp }}$ & $\Delta \tau_{\text {dtrp }}$ & $\tau_{\text {wtrp }} \Delta \tau_{\text {wtrp }}$ & $\tau_{\text {wtrp }} \Delta \tau_{\text {wtrp }}$ \\
& $(\mathrm{cm})$ & $(\mathrm{cm})$ & $(\mathrm{cm})$ & $(\mathrm{cm})$ & $(\mathrm{cm})$ & $(\mathrm{cm})$ \\
\hline Effelsberg & 220 & 0.5 & 8 & 2.7 & 20 & 6.7 \\
Hartebeesthoek & 199 & 0.5 & 10 & 3.3 & 17 & 5.7 \\
Medicina & 231 & 0.5 & 11 & 3.7 & 18 & 6.0 \\
Noto & 229 & 0.5 & 12 & 4.0 & 20 & 6.7 \\
Onsala & 230 & 0.5 & 8 & 2.7 & 14 & 4.7 \\
Sheshan & 231 & 0.5 & 22 & 7.3 & 36 & 12.0 \\
Urumqi & 210 & 0.5 & 10 & 3.3 & 10 & 3.3 \\
Westerbork & 220 & 0.5 & 8 & 2.7 & 20 & 6.7 \\
Wettzell & 215 & 0.5 & 7 & 2.3 & 13 & 4.3 \\
Yebes & 208 & 0.5 & 5 & 2.0 & 5 & 2.0 \\
\hline & & & & & &
\end{tabular}

Equivalent Flux Densities $\left(\mathrm{SEFD}_{i}\right)$ for each station according to Table 2 of the EVN Status Table ${ }^{2}$ (as available in May 2003). The Effelsberg-Westerbork baseline is the most sensitive baseline of the array but also the shortest one and so unfavorable for high-accuracy astrometry. For this reason, we decided to perform the simulations without this baseline, hence using an array of 44 baselines only. We have applied uniform weighting to the visibilities similarly to the VLBA. We have tested that in removing the 12 baselines shorter than $1500 \mathrm{~km}$ in this 44 baseline array, systematic errors decrease by $\sim 20 \%$ but, conservatively, we have kept them in our simulations. In order to reduce the number of simulations, calculations were carried out for only mean values of the wet zenith tropospheric delays since the results when using mean or maximum values were not found to be drasticaly different. We also did not calculate individual contributions from calibrator position, dry tropospheric zenith delay and Earth orientation parameter errors since these were found to be very small for the VLBA (see Tables 3 and 4). One should keep in mind, however, that calibrator error dominates at high declination. The results of the EVN simulations are reported in Tables 7 and 8 for a $1^{\circ}$ source separation in right ascension or declination.

At declination $-25^{\circ}$, many SPRINT maps were found to be ambiguous, i.e. the main lobe of the point spread function of the EVN could not be identified because secondary lobes were too high. This is essentially caused by the relatively high latitude of the array and hence to the difficulty of observing such low declination sources due to very limited visibility periods. For this reason, we do not provide EVN results for this declination. For other declinations, EVN astrometric errors (Tables 7 and 8) are similar to those found for the VLBA (Tables 3 and 4) and the Westerbork position error is not a limiting factor. Declination accuracies are somewhat better for the EVN than for the VLBA at low declination $\left(0^{\circ}\right.$ and $\left.25^{\circ}\right)$, a consequence of the participation of Hartebeeshoek (South Africa) in such observations.

\subsection{Global VLBI array}

We have carried out a similar study for the global VLBI array which is the combination of the VLBA and EVN. It includes 20 stations, with 190 possible baselines. As discussed above, the Effelsberg-Westerbork baseline was ignored and the calculations were thus carried out for 189 baselines only. The adopted

\footnotetext{
${ }^{2}$ http://www.mpifr-bonn.mpg.de/EVN/EVNstatus.txt
}

systematic error values for the simulations with this array were the same as those adopted for the individual VLBA and EVN (Tables 1,2 and 6) and calculations were performed for full track observations as previously. The results of these simulations (Tables 9 and 10) indicate that the astrometric errors for the global VLBI array are consistent with those found for the VLBA and the EVN. As expected, these errors are generally slightly better than the ones derived for each individual array.

\section{Discussion}

\subsection{General results}

Our simulations show that the astrometric accuracy of the VLBI phase-referencing technique (defined as $\sqrt{(\Delta \alpha \cos \delta)^{2}+(\Delta \delta)^{2}}$ ) is $\sim 50 \mu$ as for mid declinations and is $\leq 300 \mu$ as at low and high declinations for point sources with a relative separation of $1^{\circ}$. The major systematic error components are the wet tropospheric delay and the calibrator astrometric position, the latter only at high declination. Station coordinate, Earth orientation parameter and dry tropospheric zenith delay errors contribute generally to less than $20 \mu$ as in the error budget.

\subsection{Simulation of the VLBA without Saint Croix}

We speculated that if the VLBA station at Saint Croix in the Virgin Islands that suffers from dampness were withdrawn from the array, it should improve the astrometric accuracy of the VLBA. We thus repeated our VLBA simulations without that station. The results of this test are given in Tables 11 and 12 . In contrast to our intuition, the astrometric accuracy is actually degraded when the target-calibrator direction is oriented along declination. In fact, the addition of Saint Croix strengthens the geometry of the array and improves the astrometric accuracy despite severe weather conditions. In order to further explore this question, we ran simulations without Pie Town in the middle of the array and without Mauna Kea at the far West of the array. Withdrawing Pie Town does not change the astrometric accuracy but the absence of Mauna Kea degrades the accuracy in a similar way to Saint Croix.

\subsection{Linearity of the astrometric accuracy with source separation}

An important question is whether the astrometric accuracy scales linearly as a function of the source separation. To study this matter, we repeated all the previous simulations but with source separations of $0.5^{\circ}$ and $2^{\circ}$. Then, we performed a linear fit to the astrometric errors for the three values of the calibrator-target separation $\left(0.5^{\circ}, 1^{\circ}\right.$ and $\left.2^{\circ}\right)$, considering separately each systematic error component of the tables above. Figure 2 shows an example of such results for the VLBA in the case of a target at $+25^{\circ}$ declination. Overall, our plots show that the astrometric accuracy generally scales fairly linearly as a function of the source separation.

To obtain a quantitative measure of the likehood of the linearity, we determined the regression coefficients for each of the 107 linear fits. Such coefficients should be close to 1 for a linear behavior while they should decrease as the behavior becomes less linear. This analysis reveals that $80 \%$ of the coefficients are larger than 0.95 , indicating that the astrometric errors behave linearly. Among all errors, calibrator position systematics are those that were found to behave the least linearly. An empirical 
Table 7. EVN rms astrometric errors (in $\mu$ as) for a relative source separation $\left(\alpha-\alpha_{0}\right) \cos \delta_{0}=1^{\circ}$. Individual astrometric error contributions from station coordinate and wet troposphere uncertainties are given separately along with the total astrometric errors when all model parameters are perturbed together.

\begin{tabular}{|c|c|c|c|c|c|c|c|c|c|c|}
\hline \multirow[b]{3}{*}{ Error component } & \multicolumn{10}{|c|}{ Declination of source } \\
\hline & $0^{\circ}$ & & $25^{\circ}$ & & $50^{\circ}$ & & $75^{\circ}$ & & $85^{\circ}$ & \\
\hline & $\Delta \alpha \cos \delta$ & $\Delta \delta$ & $\Delta \alpha \cos \delta$ & $\Delta \delta$ & $\Delta \alpha \cos \delta$ & $\Delta \delta$ & $\Delta \alpha \cos \delta$ & $\Delta \delta$ & $\Delta \alpha \cos \delta$ & $\Delta \delta$ \\
\hline Antenna position & 5 & 4 & 5 & 4 & 6 & 5 & 5 & 5 & 5 & 5 \\
\hline Wet troposphere (mean) & 55 & 11 & 37 & 14 & 52 & 33 & 73 & 40 & 65 & 31 \\
\hline Total (mean wtrp) & 57 & 12 & 44 & 15 & 57 & 45 & 91 & 81 & 206 & 185 \\
\hline
\end{tabular}

Table 8. EVN rms astrometric errors (in $\mu$ as) for a relative source separation $\delta-\delta_{0}=1^{\circ}$. Individual astrometric error contributions from station coordinate and wet troposphere uncertainties are given separately along with the total astrometric errors when all model parameters are perturbed together.

\begin{tabular}{|c|c|c|c|c|c|c|c|c|c|c|}
\hline \multirow[b]{3}{*}{ Error component } & \multicolumn{10}{|c|}{ Declination of source } \\
\hline & $0^{\circ}$ & & $25^{\circ}$ & & $50^{\circ}$ & & $75^{\circ}$ & & $85^{\circ}$ & \\
\hline & $\Delta \alpha \cos \delta$ & $\Delta \delta$ & $\Delta \alpha \cos \delta$ & $\Delta \delta$ & $\Delta \alpha \cos \delta$ & $\Delta \delta$ & $\Delta \alpha \cos \delta$ & $\Delta \delta$ & $\Delta \alpha \cos \delta$ & $\Delta \delta$ \\
\hline Antenna position & 7 & 6 & 7 & 5 & 4 & 5 & 5 & 5 & 4 & 5 \\
\hline Wet troposphere (mean) & 51 & 31 & 29 & 54 & 18 & 81 & 31 & 58 & 33 & 68 \\
\hline Total (mean wtrp) & 62 & 29 & 33 & 57 & 27 & 78 & 79 & 61 & 201 & 61 \\
\hline
\end{tabular}

Table 9. Global VLBI array rms astrometric errors (in $\mu$ as) for a relative source separation $\left(\alpha-\alpha_{0}\right) \cos \delta_{0}=1^{\circ}$. The individual astrometric error contribution from wet troposphere uncertainties is given separately along with the total astrometric errors when all model parameters are perturbed together.

\begin{tabular}{|c|c|c|c|c|c|c|c|c|c|c|c|c|}
\hline \multirow[b]{3}{*}{ Error component } & \multicolumn{12}{|c|}{ Declination of source } \\
\hline & \multicolumn{2}{|l|}{$-25^{\circ}$} & \multicolumn{2}{|l|}{$0^{\circ}$} & \multicolumn{2}{|l|}{$25^{\circ}$} & \multicolumn{2}{|l|}{$50^{\circ}$} & \multicolumn{2}{|l|}{$75^{\circ}$} & \multicolumn{2}{|l|}{$85^{\circ}$} \\
\hline & $\Delta \alpha \cos \delta$ & $\Delta \delta$ & $\Delta \alpha \cos \delta$ & $\Delta \delta$ & $\Delta \alpha \cos \delta$ & $\Delta \delta$ & $\Delta \alpha \cos \delta$ & $\Delta \delta$ & $\Delta \alpha \cos \delta$ & $\Delta \delta$ & $\Delta \alpha \cos \delta$ & $\Delta \delta$ \\
\hline Wet troposphere (mean) & 71 & 76 & 32 & 42 & 26 & 34 & 23 & 13 & 22 & 6 & 27 & 9 \\
\hline Total (mean wtrp) & 82 & 67 & 34 & 46 & 24 & 44 & 34 & 33 & 64 & 76 & 196 & 203 \\
\hline
\end{tabular}

Table 10. Global VLBI array rms astrometric errors (in $\mu$ as) for a relative source separation $\delta-\delta_{0}=1^{\circ}$. The individual astrometric error contribution from wet troposphere uncertainties is given separately along with the total astrometric errors when all model parameters are perturbed together.

\begin{tabular}{|c|c|c|c|c|c|c|c|c|c|c|c|c|}
\hline \multirow[b]{3}{*}{ Error component } & \multicolumn{12}{|c|}{ Declination of source } \\
\hline & \multicolumn{2}{|c|}{$-25^{\circ}$} & \multicolumn{2}{|l|}{$0^{\circ}$} & \multicolumn{2}{|l|}{$25^{\circ}$} & \multicolumn{2}{|l|}{$50^{\circ}$} & \multicolumn{2}{|l|}{$75^{\circ}$} & \multicolumn{2}{|l|}{$85^{\circ}$} \\
\hline & $\Delta \alpha \cos \delta$ & $\Delta \delta$ & $\Delta \alpha \cos \delta$ & $\Delta \delta$ & $\Delta \alpha \cos \delta$ & $\Delta \delta$ & $\Delta \alpha \cos \delta$ & $\Delta \delta$ & $\Delta \alpha \cos \delta$ & $\Delta \delta$ & $\Delta \alpha \cos \delta$ & $\Delta \delta$ \\
\hline Wet troposphere (mean) & 60 & 305 & 26 & 71 & 10 & 43 & 9 & 44 & 5 & 21 & 5 & 26 \\
\hline Total (mean wtrp) & 61 & 279 & 24 & 78 & 15 & 45 & 22 & 46 & 61 & 24 & 183 & 27 \\
\hline
\end{tabular}

formula for the astrometric accuracy $\Delta_{\alpha} \cos \delta, \delta$ has been further estimated by averaging the parameters of all the fits:

$\Delta_{\alpha \cos \delta, \delta}=\left(\Delta_{\alpha \cos \delta, \delta}^{1^{\circ}}-14\right) \times d+14 \quad(\mu \mathrm{as})$

where $\Delta_{\alpha \cos \delta \delta}^{1^{\circ}}$ is the astrometric error for $1^{\circ}$ source separation as provided by our Tables ( 3 and 4 for the VLBA, 7 and 8 for the EVN, 9 and 10 for the global VLBI array) and $d=$ $\sqrt{\left(\left(\alpha-\alpha_{0}\right) \cos \delta_{0}\right)^{2}+\left(\delta-\delta_{0}\right)^{2}}$ is the source separation in degrees. In Sect. 4.2, we noted that the astrometric accuracies of the EVN and the VLBA are similar, hence this formula should apply to the EVN, too.

As a verification of this empirical formula, we computed the astrometric accuracy for eight target-calibrator pairs observed with the global VLBI array as part of a project to monitor absolute lobe motions in compact symmetric objects (Charlot et al. 2005). For the source pair J $2212+0152 / J 2217+0220$ with a separation of $1.37^{\circ}$ along the right ascension direction, we obtained simulated accuracies $\Delta \alpha \cos \delta_{0}=42 \mu$ as and $\Delta \delta=63 \mu$ as, versus $\Delta \alpha \cos \delta_{0}=44 \mu$ as and $\Delta \delta=63 \mu$ as when derived from Eq. (2) and Table 9. In the worst case (target-calibrator $\mathrm{J} 0754+5324 / \mathrm{J} 0753+5352$ with a separation of $0.50^{\circ}$ along declination), simulated accuracies were $\Delta \alpha \cos \delta_{0}=18 \mu$ as and $\Delta \delta=12 \mu$ as while Eq. (2) and Table 10 give $\Delta \alpha \cos \delta_{0}=20 \mu$ as and $\Delta \delta=24 \mu$ as. Thus, overall we found a discrepancy of a factor of 2 at most between our simple formula (Eq. (2)) and real simulation of the case considered.

\section{Conclusion}

We have performed extensive simulations of VLBI data with the VLBA, EVN and global VLBI array to study the dependence of the astrometric accuracy on systematic errors in the phase model of phase-referenced VLBI observations. Systematic errors considered in this study are calibrator position uncertainties, station coordinate uncertainties, Earth orientation parameters 
Table 11. VLBA without Saint Croix rms astrometric errors (in $\mu$ as) for a relative source separation $\left(\alpha-\alpha_{0}\right) \cos \delta_{0}=1^{\circ}$. The individual astrometric error contribution from wet troposphere uncertainties is given separately along with the total astrometric errors when all model parameters are perturbed together.

\begin{tabular}{|c|c|c|c|c|c|c|c|c|c|c|c|c|}
\hline \multirow[b]{3}{*}{ Error component } & \multicolumn{12}{|c|}{ Declination of source } \\
\hline & \multicolumn{2}{|c|}{$-25^{\circ}$} & \multicolumn{2}{|l|}{$0^{\circ}$} & \multicolumn{2}{|l|}{$25^{\circ}$} & \multicolumn{2}{|l|}{$50^{\circ}$} & \multicolumn{2}{|l|}{$75^{\circ}$} & \multicolumn{2}{|l|}{$85^{\circ}$} \\
\hline & $\Delta \alpha \cos \delta$ & $\Delta \delta$ & $\Delta \alpha \cos \delta$ & $\Delta \delta$ & $\Delta \alpha \cos \delta$ & $\Delta \delta$ & $\Delta \alpha \cos \delta$ & $\Delta \delta$ & $\Delta \alpha \cos \delta$ & $\Delta \delta$ & $\Delta \alpha \cos \delta$ & $\Delta \delta$ \\
\hline Wet troposphere (mean) & 62 & 171 & 28 & 47 & 27 & 57 & 34 & 65 & 46 & 63 & 37 & 67 \\
\hline Total (mean wtrp) & 63 & 193 & 27 & 41 & 31 & 62 & 42 & 68 & 83 & 82 & 211 & 207 \\
\hline
\end{tabular}

Table 12. VLBA without Saint Croix rms astrometric errors (in $\mu$ as) for a relative source separation $\delta-\delta_{0}=1^{\circ}$. The individual astrometric error contribution from wet troposphere uncertainties is given separately along with the total astrometric errors when all model parameters are perturbed together.

\begin{tabular}{|c|c|c|c|c|c|c|c|c|c|c|c|c|}
\hline \multirow[b]{3}{*}{ Error component } & \multicolumn{12}{|c|}{ Declination of source } \\
\hline & \multicolumn{2}{|c|}{$-25^{\circ}$} & \multicolumn{2}{|l|}{$0^{\circ}$} & \multicolumn{2}{|l|}{$25^{\circ}$} & \multicolumn{2}{|l|}{$50^{\circ}$} & \multicolumn{2}{|l|}{$75^{\circ}$} & \multicolumn{2}{|l|}{$85^{\circ}$} \\
\hline & $\Delta \alpha \cos \delta$ & $\Delta \delta$ & $\Delta \alpha \cos \delta$ & $\Delta \delta$ & $\Delta \alpha \cos \delta$ & $\Delta \delta$ & $\Delta \alpha \cos \delta$ & $\Delta \delta$ & $\Delta \alpha \cos \delta$ & $\Delta \delta$ & $\Delta \alpha \cos \delta$ & $\Delta \delta$ \\
\hline Wet troposphere (mean) & 183 & 563 & 62 & 189 & 19 & 55 & 17 & 41 & 39 & 27 & 42 & 44 \\
\hline Total (mean wtrp) & 190 & 534 & 70 & 191 & 24 & 71 & 26 & 42 & 74 & 28 & 216 & 40 \\
\hline
\end{tabular}
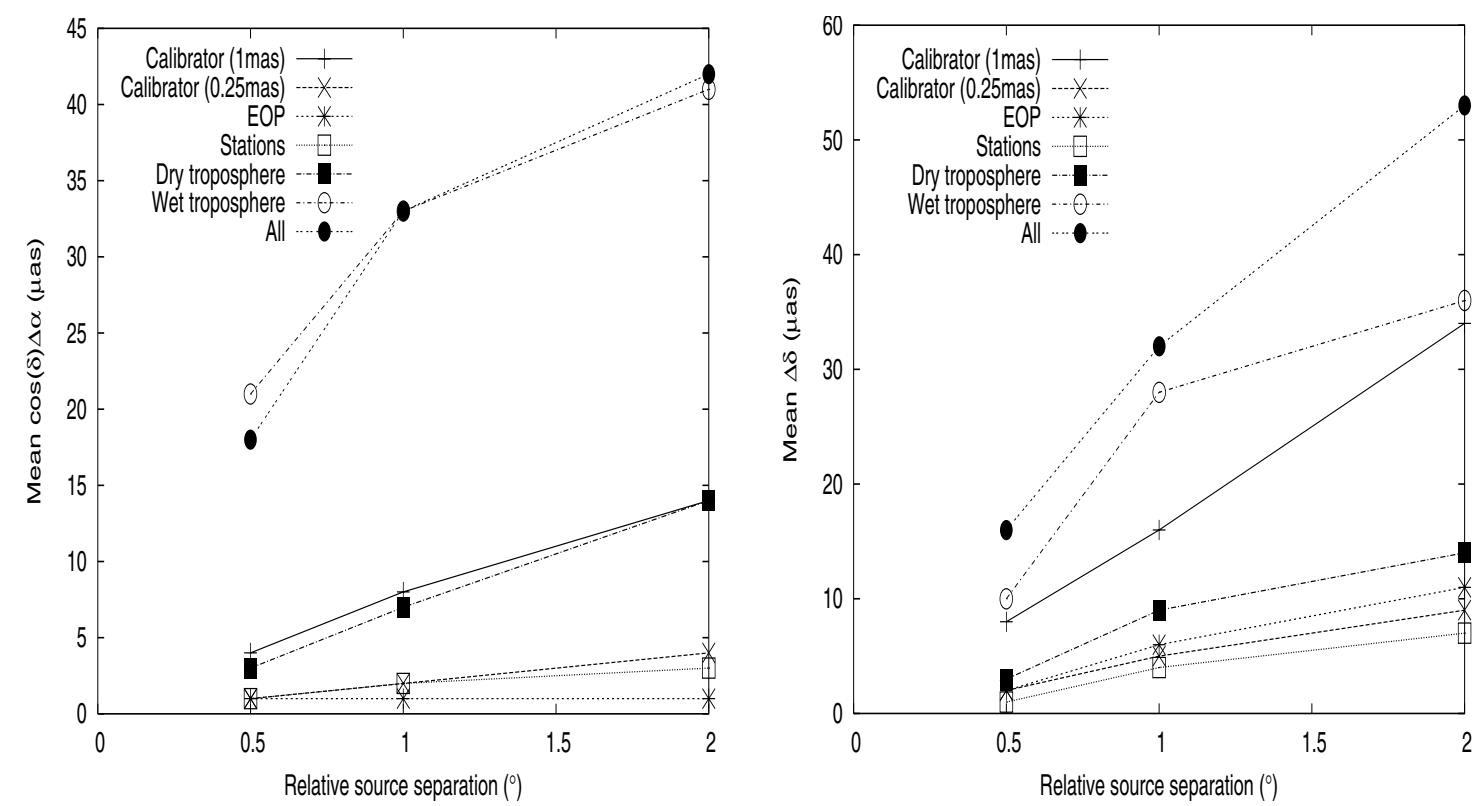

Fig. 2. Astrometric accuracy as a function of the relative source separation for a target-calibrator pair observed with the VLBA at a declination of $+25^{\circ}$. Each error component is represented with a different symbol and the total contributing error is also plotted.

uncertainties and dry and wet troposphere errors. We have adopted state of the art VLBI values for these errors.

Our simulations show that the astrometric accuracy of a full track phase-referenced VLBI experiment is $50 \mu$ as at mid declination and is $\sim 300 \mu$ as at low $\left(-25^{\circ}\right)$ and high $\left(+85^{\circ}\right)$ declinations for point sources angularly separated by $1^{\circ}$. Not surprinsingly, the major systematic error originates from wet tropospheric zenith delay uncertainties except at high declination where calibrator position uncertainties dominate. We show that the astrometric accuracy $\Delta_{\alpha \cos \delta, \delta}$ depends linearly on the source separation and we established the simple formula $\Delta_{\alpha \cos \delta, \delta}=$ $\left(\Delta_{\alpha \cos \delta, \delta}^{1^{\circ}}-14\right) \times d+14$ ( $\mu$ as) where $\Delta_{\alpha \cos \delta, \delta}^{1^{\circ}}$ is the astrometric error provided by our tables for the various arrays and configurations and $d=\sqrt{\left(\left(\alpha-\alpha_{0}\right) \cos \delta\right)^{2}+\left(\delta-\delta_{0}\right)^{2}}$ is the source separation in degrees. Our study has been carried out for point sources but variable source structure is likely to degrade the accuracy derived from this formula.

\section{Appendix A: Analytical behavior}

The analytical formulae in the Appendix A of Shapiro et al. (1979) provide the astrometric errors caused by the inaccuracy of the calibrator coordinates in the case of a single VLBI baseline. Adopting our notation, these formulae become:

$\Delta \alpha \simeq\left(\left(\alpha-\alpha_{0}\right) \tan \delta\right) \Delta \delta_{0}$

$$
-\left(\left(\delta-\delta_{0}\right) \tan \delta+1 / 2 \times\left(\alpha-\alpha_{0}\right)^{2}\right) \Delta \alpha_{0},
$$

and

$$
\begin{aligned}
\Delta \delta \simeq & \left(-\left(\delta-\delta_{0}\right) \cot \delta+1 / 2 \times\left(\alpha-\alpha_{0}\right)^{2}\right) \Delta \delta_{0} \\
& +\left(\left(\alpha-\alpha_{0}\right) \cot \delta\right) \Delta \alpha_{0},
\end{aligned}
$$

where $\Delta \alpha$ and $\Delta \delta$ are the errors in right ascension and declination introduced by errors $\Delta \alpha_{0}$ and $\Delta \delta_{0}$ in the coordinates of the reference source. The expression above for $\Delta \delta$ restores correctly 



Fig. A.1. Astrometric errors $\Delta \alpha \cos \delta$ and $\Delta \delta$ (respectively left and right) as a function of declination. The two upper plots are for the case $\alpha-\alpha_{0}=$ $(1 / \cos \delta)^{\circ}$ and $\delta-\delta_{0}=0^{\circ}$ while the two lower plots are for the case $\alpha-\alpha_{0}=0^{\circ}$ and $\delta-\delta_{0}=1^{\circ}$. The continuous dotted lines show the errors derived from the Shapiro et al. (1979) formulae. The stars show the errors from our simulations at six declinations from $-25^{\circ}$ to $85^{\circ}$.

the last term of the equation which was misprinted in the original paper. These simple formulae are, however, valid only for the special geometry adopted by the authors where the "baseline declination" is $0^{\circ}$.

Adopting the same parameters as in our simulations $\left(\Delta \alpha_{0}=\right.$ $1 / \cos \delta_{0}$ mas, $\Delta \delta_{0}=1$ mas, $\alpha-\alpha_{0}=0^{\circ}$ or $\left(1 / \cos \delta_{0}\right)^{\circ}$, $\delta-\delta_{0}=1^{\circ}$ or $0^{\circ}$ ), we obtain the astrometric errors plotted as a function of declination in Fig. A.1 (dotted lines). The results of our simulations for declinations of $-25^{\circ}, 0^{\circ}, 25^{\circ}, 50^{\circ}, 75^{\circ}$ and $85^{\circ}$ in the case of the VLBA (first lines of Tables 3 and 4) are also superimposed on these plots.

The right ascension errors obtained from the simulations match perfectly those derived analytically, while the declination errors show a strong discrepancy near declination $0^{\circ}$ (although they agree at high declinations). This discrepancy originates from a singularity in the $\Delta \delta$ formula at $\delta=0^{\circ}$ (term in $\cot \delta$ ), inherent to the approximation used to establish the formula (baseline declination of $0^{\circ}$ ). For a more complex and realistic network, such a singularity does not exist, as also demonstrated by the results of our simulations.

\section{References}

Beasley, A. J., Gordon, D., Peck, A. B., et al. 2002, ApJS, 141, 13

Boucher, C., Altamimi, Z., Sillard, P., \& Feissel-Vernier, M. 2004, The ITRF 2000, IERS Technical Note No. 31, IERS, Verlag des Bundesamts für Kartographie und Geodäsie, Frankfurt am Main

Brisken, W. F., Benson, J. M., Goss, W. M., \& Thorsett, S. E. 2002, ApJ, 571, 906

Carilli, C. L., \& Holdaway, M. A. 1999, Radio Science, 34, 817

Charlot, P. 1990, AJ, 99, 1309

Charlot, P., Campbell, R. M., Alef, W., et al. 2002, in Proc. of the 6th European VLBI Network Symposium, ed. E. Ros, R. W. Porcas, A. P. Lobanov, \& J. A. Zensus (Bonn: MPIfR), 9

Charlot, P., Pradel, N., \& Lestrade, J.-F. 2005, in Future Directions in High Resolution Astronomy, ed. J. D. Romney, \& M. J. Reid, ASP Conf. Ser., 340, 511

Fomalont, E. B., Goss, W. M., Beasley, A. J., \& Chatterjee, S. 1999, AJ, 117, 3025

Honma, M., Kawaguchi, N., \& Sasao, T. 2000, in Proc. SPIE Radio Telescopes, ed. H. R. Butcher, 4015, 624

Kawaguchi, N., Sasao, T., \& Manabe, S. 2000, in Radio Telescopes, ed. H. R. Butcher, Proc. SPIE, 4015, 544

Lebach, D. E., Ratner, M. I., Shapiro, I. I., et al. 1999, ApJ, 517, L43

Lestrade, J.-F., Jones, D. L., Preston, R. A., \& Phillips, R. B. 1994, Ap\&SS, 212, 251

Lestrade, J.-F., Preston, R. A., Jones, D. L., et al. 1999, A\&A, 344, 1014

Lestrade, J.-F., Rogers, A. E. E., Whitney, A. R., et al. 1990, AJ, 99, 1663

Ly, C., Walker, R. C., \& Wrobel, J. M. 2004, AJ, 127, 119

Ma, C., Arias, E. F., Eubanks, T. M., et al. 1998, AJ, 116, 516

Marcaide, J. M., \& Shapiro, I. I. 1983, AJ, 88, 1133

Marcaide, J. M., \& Shapiro, I. I. 1984, ApJ, 276, 56

Morabito, D. D. 1984, Telecommunications and Data Acquisition Progress Report, 79, 1

Reid, M. J., Menten, K. M., Genzel, R., et al. 2003, ApJ, 587, 208

Ros, E., Marcaide, J. M., Guirado, J. C., et al. 1999, A\&A, 348, 381

Saastamoinen, J. 1973, Bulletin Géodésique, 105, 279

Shapiro, I. I., Wittels, J. J., Counselman, C. C., et al. 1979, AJ, 84, 1459

Sovers, O. J. 2003, private communication

Sovers, O. J., Fanselow, J. L., \& Jacobs, C. S. 1998, Rev. Mod. Phys., 70, 1393

Thompson, A. R., Moran, J. M., \& Swenson, G. W. 1986, Interferometry and Synthesis in Radio Astronomy (New York: Wiley-Interscience) 